\title{
Ultrafast Polariton-Phonon Dynamics of Strongly Coupled Quantum Dot-Nanocavity Systems
}

\author{
Kai Müller, Kevin A. Fischer, Armand Rundquist, Constantin Dory, Konstantinos G. Lagoudakis, \\ Tomas Sarmiento, Yousif A. Kelaita, Victoria Borish, and Jelena Vučković \\ E. L. Ginzton Laboratory, Stanford University, Stanford, California 94305, USA
}

(Received 18 March 2015; revised manuscript received 26 May 2015; published 16 July 2015)

\begin{abstract}
We investigate the influence of exciton-phonon coupling on the dynamics of a strongly coupled quantum dot-photonic crystal cavity system and explore the effects of this interaction on different schemes for nonclassical light generation. By performing time-resolved measurements, we map out the detuningdependent polariton lifetime and extract the spectrum of the polariton-to-phonon coupling with unprecedented precision. Photon-blockade experiments for different pulse-length and detuning conditions (supported by quantum optical simulations) reveal that achieving high-fidelity photon blockade requires an intricate understanding of the phonons' influence on the system dynamics. Finally, we achieve direct coherent control of the polariton states of a strongly coupled system and demonstrate that their efficient coupling to phonons can be exploited for novel concepts in high-fidelity single-photon generation.
\end{abstract}

DOI: 10.1103/PhysRevX.5.031006

\section{INTRODUCTION}

The strong coupling between a single photon and single quantum emitter is of substantial interest for both investigations of the fundamentals of quantum optics and potential applications in optical computing, quantum metrology, and quantum cryptography [1-3]. This universality is reflected by the diversity of associated experimental realizations, ranging from those in atomic physics [4-6] to superconducting systems $[7,8]$ and semiconductor devices [9]. In the solid state, self-assembled quantum dots (QDs) are the most investigated quantum emitters because of their strong interaction with light as well as their nearly transform-limited linewidth [10-12]. Optical resonators, in the form of both micropillar structures [9] and photonic crystal cavities [13], are widely used to enhance this lightmatter interaction. Photonic crystal cavities are especially promising for on-chip integration of quantum optical circuits because of the convenient fabrication of integrated waveguide and detector structures [14]. In contrast to other systems, semiconductor quantum emitters are usually embedded in a crystalline host matrix, resulting in strong interactions with phonons. While in many circumstances such interactions are undesirable, it is also possible to carefully design experiments that benefit from the presence of phonons. For example, it was recently demonstrated that

\footnotetext{
*kaim@stanford.edu
}

Published by the American Physical Society under the terms of the Creative Commons Attribution 3.0 License. Further distribution of this work must maintain attribution to the author(s) and the published article's title, journal citation, and DOI.
Subject Areas: Condensed Matter Physics,

Photonics, Quantum Physics the QD coupling to phonons can be exploited for a robust and high-fidelity preparation of exciton and biexciton states [15-17]. Meanwhile, when QDs are coupled to semiconductor optical cavities, far off-resonant feeding effects have been shown to be consistent with a phononinduced interaction [18,19]. In fact, for QD-cavity systems, the coupling to phonons and its impact on applications has been extensively studied in the weakly coupled regime $[20,21]$. However, for strongly coupled systems, these effects remain largely unexplored experimentally. As such systems become increasingly relevant for their potential in building quantum optical networks, it is essential to develop a solid understanding of the influence that phonons have on the system dynamics.

In this paper, we investigate the interaction of a strongly coupled QD-cavity system with phonons and explore the impact of this coupling on different schemes for nonclassical light generation. By performing temporally resolved spectroscopy experiments, we map out the detuning-dependent polariton lifetime and extract the spectrum of the polaritonto-phonon coupling. As a result, we conclude that the high efficiency of this coupling entails its importance for all applications of strongly coupled solid-state systems. To corroborate this finding, we investigate the influence of electron-phonon interaction on one of the key applications of strongly coupled systems: nonclassical light generation. To this end, we perform photon-blockade experiments and complementary quantum optical simulations under different pulse-length and detuning conditions, which reveal that the pulse length has to be chosen correctly for a given detuning in order to obtain a high-fidelity photon blockade. In particular, the impact of coupling to phonons is found to 
be more pronounced for the case of a detuned photon blockade, which is the condition that yields the highest fidelity and efficiency [22]. Finally, we demonstrate that the efficient coupling to phonons can also be utilized for novel concepts in high-fidelity single-photon generation.

\section{STRONGLY COUPLED QD-CAVITY SYSTEMS}

The sample investigated consists of a layer of lowdensity InAs QDs grown by molecular beam epitaxy and embedded in a photonic crystal L3 cavity [23]. The energy structure of a QD strongly coupled to an optical cavity (without phonon coupling) is usually described by the Jaynes-Cummings (JC) Hamiltonian

$$
H=\omega_{a} a^{\dagger} a+\left(\omega_{a}+\Delta\right) \sigma^{\dagger} \sigma+g\left(a^{\dagger} \sigma+a \sigma^{\dagger}\right),
$$

where $\omega_{a}$ denotes the frequency of the cavity, $a$ the cavity mode operator, $\sigma$ the lowering operator of the quantum emitter, $\Delta$ the detuning between quantum emitter and cavity, and $g$ the emitter-cavity field coupling strength. After introducing dissipation into the JC system, we obtain a quantum Liouville equation with complex eigenenergies [24]:

$$
\begin{aligned}
E_{ \pm}^{n}= & n \omega_{a}+\frac{\Delta}{2}-i \frac{(2 n-1) \kappa+\gamma}{4} \\
& \pm \sqrt{(\sqrt{n} g)^{2}+\left(-\frac{\Delta}{2}-i \frac{\kappa-\gamma}{4}\right)^{2}}
\end{aligned}
$$

where $E_{ \pm}^{n}$ corresponds to the $n$th rung of the system and $\kappa$ and $\gamma$ to the cavity and QD energy decay rates, respectively. The real parts of $E_{ \pm}^{n}$ yield the energies of the states, whereas the imaginary parts yield their linewidths. Note that all four parameters $\kappa, \gamma, \Delta$, and $g$ contribute to the polariton splittings and linewidths.

The resulting eigenenergies, the Jaynes-Cummings-ladder dressed states, are presented in Fig. 1(a). They consist of a series of anticrossing branches that are labeled UPn (LP $n$ ) for the upper (lower) polariton, where $n$ is the index of the rung. For $n$ photons in the cavity, the energy is $n \omega_{a}$ (dashed red lines), and the energy of the quantum emitter (dotted green line) varies with a detuning parameter. Because of the coupling, the resulting energy eigenstates are the anticrossing polariton branches. At resonance, the splitting is given by $2 g \sqrt{n}$.

Experimentally, we can observe the splitting of the lowest-manifold polaritons in cross-polarized reflectivity [25] through control of the crystal lattice temperature. The result of such an experiment is presented in Fig. 1(b) and shows a clear anticrossing. A fit to the data reveals values of $g=12.3 \cdot 2 \pi \mathrm{GHz}$ and $\kappa=18.4 \cdot 2 \pi \mathrm{GHz}$. The radiative lifetime of a QD in a bulk photonic environment is known to be about $1 \mathrm{~ns}$, and thus, for QDs in photonic
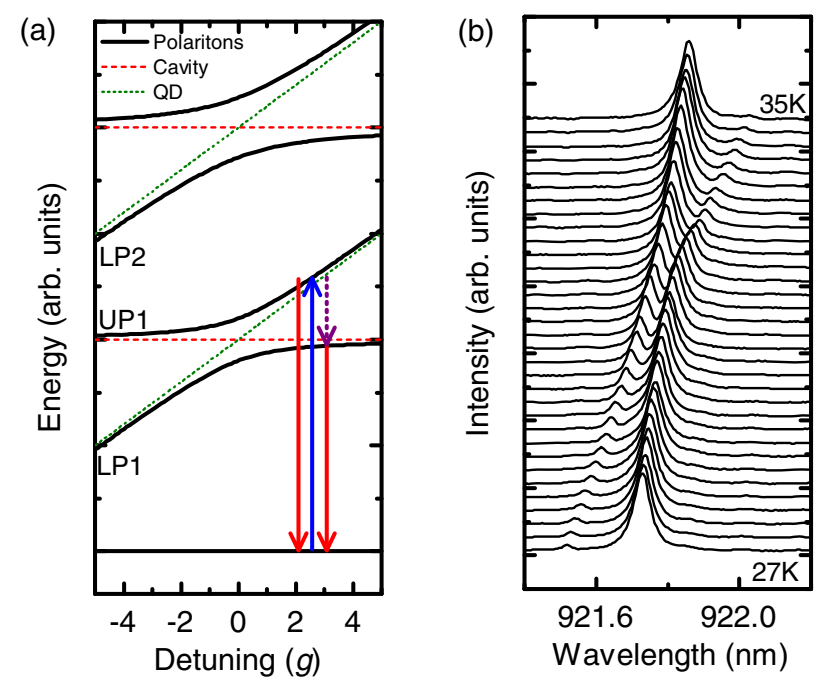

FIG. 1. (a) Schematic illustration of the energy structure of a strongly coupled system as given by the Jaynes-Cummings ladder obtained from Eq. (2). The blue arrow illustrates resonant excitation of UP1, the red arrows show photon decay, and the purple arrow is the phonon-assisted transfer. (b) Cross-polarized reflectivity spectra of the coupled QD-cavity system obtained by temperature tuning the QD through the cavity resonance. An anticrossing of the peaks clearly demonstrates the strong coherent coupling.

crystal cavities, $\gamma$ is much smaller than $\kappa$. Because of the photonic band gap, $\gamma$ is further suppressed, and reported values for the continuum mode lifetimes are around $4-12 \mathrm{~ns}[26,27]$ corresponding to $\gamma \sim 0.01 \cdot 2 \pi \mathrm{GHz}$.

\section{POLARITON DECAY RATES}

Using the values above and Eq. (2), we plot the decay rates (imaginary parts of $E_{ \pm}^{n}$ ) and radiative lifetimes of the polariton branches of an ideal Jaynes-Cummings system as a function of the detuning in Figs. 2(a) and 2(b), respectively. In these plots, the red (blue) curve corresponds to UP1 (LP1), which is more cavity-like (QD-like) for negative and QD-like (cavity-like) for positive detunings. The decay rates and thus radiative lifetimes are exchanged over a range that is determined by $g$ and $\kappa$.

In contrast to ideal JC systems, however, for solid-state systems a phonon-assisted population transfer between QD excitons and cavity photons is known to exist [20,28-30]. Thus, we sought to determine the true state lifetimes through time-resolved measurements. The fast cavity decay rate and strong interaction with phonons are expected to result in significantly shortened lifetimes. Hence, we performed the measurements using pulsed resonant excitation and time-resolved detection on a streak camera with sub-5-ps resolution. While nonresonant excitation would lead to slow carrier relaxation into the QDs [31], our resonant scheme minimizes timing jitter in the excitation. To avoid exciting higher rungs of the JC ladder, we pumped the more QD-like polariton branch UP1 [as schematically 

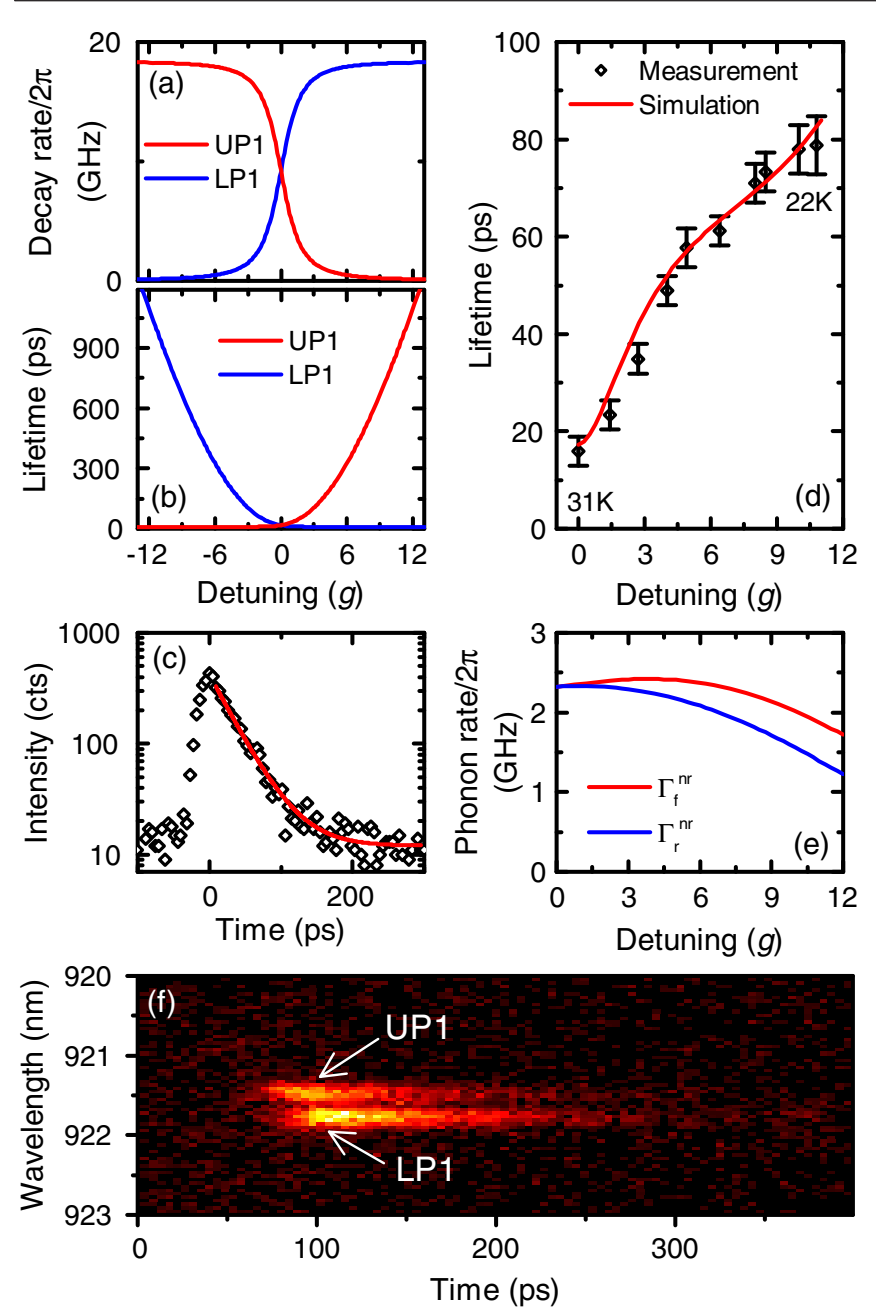

FIG. 2. (a) Decay rates and (b) resulting lifetimes of the polariton branches as functions of the QD-cavity detuning, obtained from Eq. (2). (c) Time-resolved spectrally integrated luminescence intensity at a detuning of $\Delta=2.8 \mathrm{~g}$ for excitation of UP1. The lifetime is extracted from a mono-exponential fit (shown in red). (d) Measured and simulated lifetime of UP1 as a function of the detuning. (e) Phonon-assisted state transfer rates extracted from the lifetime at each detuning. (f) Spectrally and temporally resolved luminescence at a QD-cavity detuning of $\Delta=7 g$. The delayed onset of emission from LP1 for resonant excitation of UP1 is consistent with phonon-assisted population transfer.

illustrated by the blue arrow on the right side of Fig. 1(a)] with a pulse length that was carefully chosen to be shorter than the state lifetime but spectrally sharp enough not to overlap with the other polariton branch. Luminescence was then observed either from this state directly [red arrow in Fig. 1(a)] or from the more cavity-like polariton branch after a phonon-assisted transfer [purple and red arrows in Fig. 1(a)]. The dynamics of this process can be simulated using a rate equation model with four rates: radiative recombination from UP1 $\left(\Gamma_{U P 1}^{r}\right)$, radiative recombination from LP1 $\left(\Gamma_{L P 1}^{r}\right)$, the phonon-assisted transfer rate from UP1 to LP1 $\left(\Gamma_{f}^{n r}\right)$, and vice versa $\left(\Gamma_{r}^{n r}\right)$. As shown in

Fig. 2(c), the spectrally integrated photoluminescence intensity of a typical time-resolved measurement at a QD-cavity detuning of $\Delta=2.8 g$ (including contributions from both decay paths) shows a mono-exponential decay, as expected from the rate equation model. A fit to the data [red line in Fig. 2(c)] reveals a decay time of $34.9 \pm 2$ ps. We note here that the spectrally integrated intensity decays with the same time constant as the UP1 line (see Supplemental Material Ref. [32] for details on the rate equation model).

To map out the polariton lifetimes, we repeated the timeresolved measurement presented in Fig. 2(c) for different detunings in the range $\Delta=0-11 \mathrm{~g}$. As shown in Fig. 2(d), the resulting lifetime of UP1 increases with increasing detuning. However, the increase occurs much more slowly than indicated by the rates calculated from the JC model assuming no phonon interaction, as presented in Fig. 2(b). For the detunings investigated here, LP1 is more cavity-like and, thus, has a much shorter radiative lifetime, ranging from $16 \mathrm{ps}$ down to $8 \mathrm{ps}$ as the detuning is increased. Meanwhile, the JC lifetime without phonon interaction for UP1 varies from 16 ps up to almost $1000 \mathrm{ps,} \mathrm{while} \mathrm{the}$ measured lifetime of UP1 only increases up to roughly 80 ps. Therefore, a strong phonon-assisted population transfer from UP1 to LP1 significantly shortens the observed lifetime of UP1.

We model this phonon-assisted population transfer using an effective master equation derived in a polaron frame with respect to the phonon interaction [30]. This model fully captures the polariton dynamics and correlations discussed here without the need for non-Markovian damping. Additionally, the model is valid for our system over a wide range of temperatures, even extending to near absolute zero (see Supplemental Material Ref. [32] for details on the model and its validity). A fit to the data including this model is presented as a red line in Fig. 2(d) and produces very good overall agreement with all of the measured data. Since the detuning between the cavity and QD was controlled by the lattice temperature, the relative temperature of each detuning was taken into account for these simulations. From the fit, we can extract the phononassisted population transfer rates $\Gamma_{f}^{n r}$ and $\Gamma_{r}^{n r}$. They are presented in Fig. 2(e) as red and blue lines, respectively. Near resonance, the two rates are very close because of the elevated temperature of $T=31 \mathrm{~K}$. With increasing detuning, both rates decrease as the polariton states evolve towards the bare QD and cavity states, where the phonon interaction cannot couple polaritons through the cavity drive. The lower values of $\Gamma_{r}^{n r}$ compared to $\Gamma_{f}^{n r}$ result from the different probabilities of phonon emission and absorption at low temperatures.

In order to further corroborate this model of phononassisted population transfer, we performed measurements with simultaneous spectral and temporal resolution. The result of a typical measurement recorded at $\Delta=7 \mathrm{~g}$ is 
presented in Fig. 2(f). Two distinct luminescence peaks are visible. Most importantly, the onset of luminescence from LP1 is delayed with respect to the onset of luminescence from UP1, as population must be transferred before photons can be emitted. At this detuning, the radiative recombination rate $\Gamma_{U P 1}^{r}$ is small compared to the strong phonon-assisted population transfer and subsequent radiative recombination of LP1 $\left(\Gamma_{L P 1}^{r}\right)$. Hence, the strongest luminescence intensity is observed from LP1 even though UP1 is resonantly excited (for details, see Supplemental Material Ref. [32]).

\section{PULSE-LENGTH-DEPENDENT PHOTON BLOCKADE}

Having mapped out the detuning-dependent polariton lifetimes of the strongly coupled QD-cavity system, we can now apply this knowledge to applications where the lifetime is critical. One of the most remarkable applications of strongly coupled systems is the photon blockade $[1,35]$, in which a laser pulse is tuned to the first rung of the JC ladder and (because of the anharmonicity) is not resonant with higher transitions up the ladder. The JC system then transmits the portion of the incident pulse that couples to the system, which we observe in our cross-polarized reflectivity measurements. Therefore, the probability of coupling a single photon through the system is enhanced over that of multiphoton states, which results in a transmitted light beam that has a strong nonclassical character. The fidelity of this process for the generation of single photons is inherently limited due to the linewidth of the polariton branches. Nevertheless, it was recently shown that in a strongly coupled system that is detuned by a few $g$, not only the purity but also the efficiency of single-photon generation increases [22]. As we will see, the photon blockade depends crucially on the polariton lifetimes, especially in the detuned case.

To quantify the nonclassical character of the photocount distribution, we use its measured degree of second-order coherence for zero time delay [36,37],

$$
g^{(2)}(0)=\frac{\langle n(n-1)\rangle}{\langle n\rangle^{2}},
$$

computed from expectations of the measured photocount distribution, where $n$ signifies the number of detections. Only nonclassical light sources may have a second-order coherence $g^{(2)}(0)<1$, and $g^{(2)}(0)=0$ is measured exclusively for pulses with single-photon-like character. Owing to a cavity lifetime much shorter than the timing jitter of the single photon counters, measurements of $g^{(2)}(0)$ on our system can only be performed under pulsed excitation; this is in any case the experimental configuration required for on-demand applications. Hence, the choice of pulse length forces a compromise between frequency resolution (reducing the overlap of different rungs) and the likelihood of reexcitation of the system. In other words, if the laser pulses are too long, the system will be reexcited during the interaction with a single pulse, reducing the nonclassical character of the transmitted light. On the other hand, pulses with a shorter duration are spectrally broader, resulting in a larger overlap with higher rungs. With increasing detuning between QD and cavity resonances, the lifetime of the emitter-like (cavity-like) polariton branch increases (decreases). Therefore, in order to obtain the strongest photon blockade, the pulse length has to be chosen according to the detuning-dependent polariton lifetime.

To test this hypothesis, we performed measurements of $g^{(2)}(0)$ using a Hanbury Brown and Twiss (HBT)-type experiment [38] for different QD-cavity detunings (and thus polariton lifetimes) and pulse lengths. Figure 3(a) shows $g^{(2)}(0)$ as a function of the laser detuning for pulse lengths [39] of $34 \mathrm{ps}$ on the left and $110 \mathrm{ps}$ on the right, and detunings of $\Delta=0 g, \Delta=2 g$, and $\Delta=4 g$ from top to bottom. At $\Delta=0$, both pulse lengths result in a symmetric curve with photon-blockade dips of $g^{(2)}(0)<1$ for laser detunings of $1-2 g$. We measure a minimum $g^{(2)}(0)=$ $0.88 \pm 0.03$ for 34 -ps pulses and $g^{(2)}(0)=0.93 \pm 0.03$ for 110-ps pulses. Because of the short polariton lifetime of 16 ps at $\Delta=0$, the longer pulses result in an increased likelihood of reexcitation during the presence of a single pulse, which leads to a higher value of $g^{(2)}(0)$ in the photon blockade. We note that since the radiative recombination rates are very fast when the QD and the cavity are in resonance, phonons have only a minor effect in this case (see Supplemental Material Ref. [32] for simulations and details).

With increasing detuning, the fidelity of the photon blockade increases as expected [22], and for $\Delta=4 \mathrm{~g}$ [bottom plots of Fig. 3(a)], we measure values as low as $g^{(2)}(0)=0.44 \pm 0.07$ for 34-ps pulses and $g^{(2)}(0)=$ $0.34 \pm 0.07$ for 110 -ps pulses. In particular, because of the longer polariton lifetime at $\Delta=4 g$, we observe a lower value of $g^{(2)}(0)$ for the longer pulses. Here, reexcitation limits the minimum of the second-order coherence for the long pulses, while spectral overlap with transitions to higher rungs limits the minimum for the short pulses.

To explain this finding, we performed quantum optical simulations using the Quantum Optics Toolbox in Python (QuTiP) [40] based on the quantum regression theorem [22]. The results of these simulations are presented in Figs. 3(b)-3(c). The figures show $g^{(2)}(0)$ as a function of the laser detuning and pulse length for $\Delta=4 \mathrm{~g}$, both including [Fig. 3(b)] and excluding [Fig. 3(c)] phononassisted population transfer (see Supplemental Material Ref. [32] for details on the simulations and additional representations). In both cases, with increasing pulse length, the photon-blockade dip narrows down (as seen in the experiment) because of the improved spectral resolution of longer pulses. Moreover, the minimum value of $g^{(2)}(0)$ decreases because of the decreased overlap with 


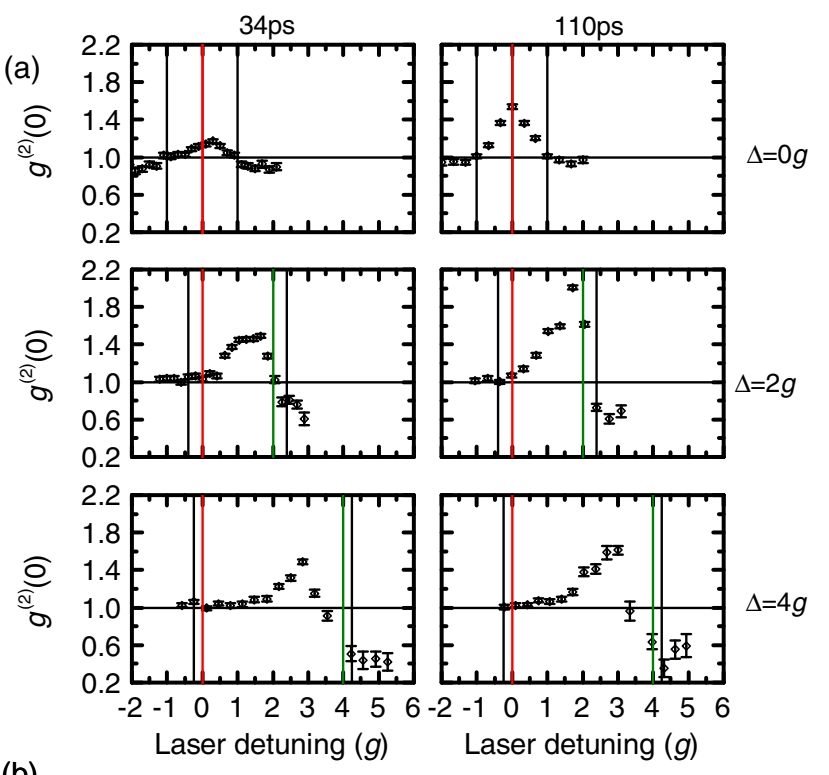

(b)
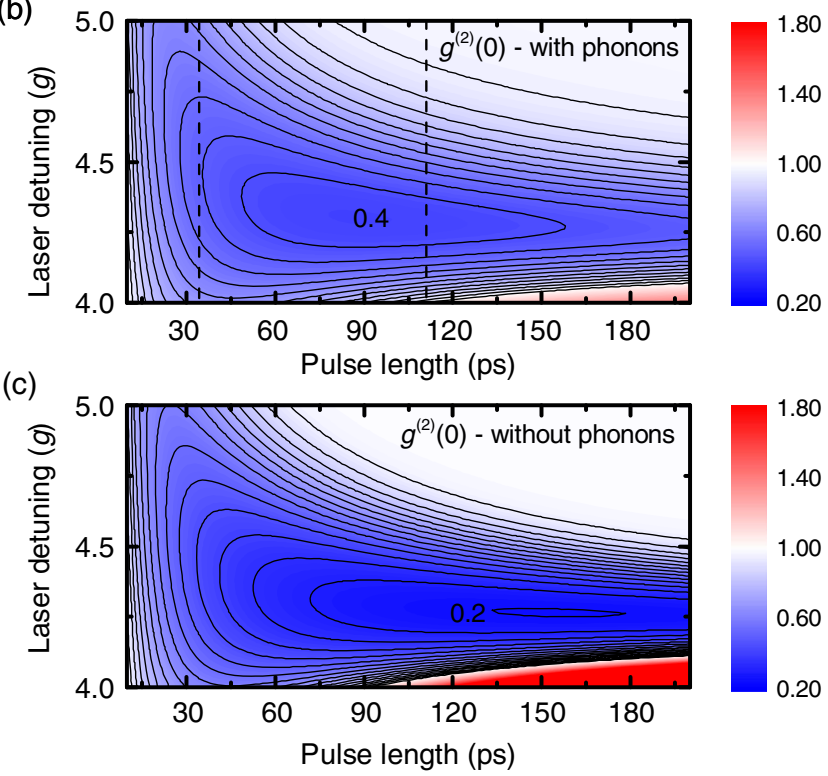

FIG. 3. (a) Measured second-order coherence $g^{(2)}(0)$ as a function of the laser detuning for different QD-cavity detunings (top to bottom) and excitation pulse lengths (left to right). In each case, the red vertical line indicates the bare cavity frequency, the green vertical line indicates the bare QD frequency, and the black vertical lines indicate the polariton frequencies. (b-c) Simulated photon-blockade values of $g^{(2)}(0)$ as a function of the laser detuning and pulse length including (b) and excluding (c) the effect of phonons, both at a QD-cavity detuning of $\Delta=4 g$. To aid in comparison with the experimental results in (a), the dashed lines in (b) indicate the simulated antibunching values for the photon blockade at the experimental pulse lengths.

higher rungs (as well as the other polaritonic branch) before increasing from reexcitation for too-long pulses. As can be seen by comparing Fig. 3(b) with Fig. 3(c), the phononinduced reduction of the polariton lifetime means that the deepest photon-blockade dip is observed at a shorter pulse length when phonons are included, and the corresponding minimum $g^{(2)}(0)$ values are a little higher. The simulations are in very good overall agreement with the measurements presented in Fig. 3(a), and they highlight the importance of taking phonon-assisted transfer into account when selecting the optimal pulse length.

For an excitation between the polariton branches, a peak with $g^{(2)}(0)>1$ is observed for all detunings and pulse lengths. This phenomenon is known as photon-induced tunneling [1], where anharmonicity of the JC ladder results in bunching. However, the bunching values peak at the position where the probability of zero detections is highest and simulations of the photodetection distributions barely deviate from those of a coherent state with a similar count rate [22]. Therefore, in this paper, we focus on the antibunching regime.

\section{COHERENTLY EXCITED SINGLE-PHOTON SOURCE}

While we have seen above that for a detuned photon blockade the presence of exciton-phonon coupling slightly decreases the fidelity, this coupling also allows us to investigate more sophisticated schemes for on-demand single-photon generation. In particular, the population of UP1 can be coherently controlled if the pulse length is chosen to be shorter than the state lifetime but spectrally narrow enough to avoid exciting higher transitions up the JC ladder. Because of coherent scattering of the laser and imperfect suppression of the laser reflection from the sample surface, it is difficult to observe Rabi oscillations directly. However, phonon-assisted emission from LP1 following excitation of UP1 occurs at a different frequency. In addition, for detunings in the range of $\Delta=5-10 g, \Gamma_{U P 1}^{r}$ is strongly Purcell suppressed while $\Gamma_{L P 1}^{r}$ is very fast. Most strikingly, $\Gamma_{f}^{n r}$ is proportional to $g^{2}$ [30] and, thus, very efficient for a strongly coupled system. Therefore, even when resonantly exciting UP1 for these detunings, most luminescence is emitted from LP1. The result of such an experiment is presented in Fig. 4(a), which shows the emission intensity from LP1 for a resonant excitation of (a)

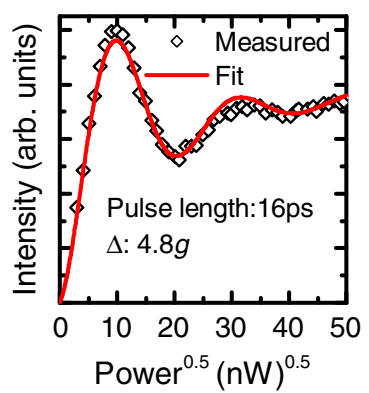

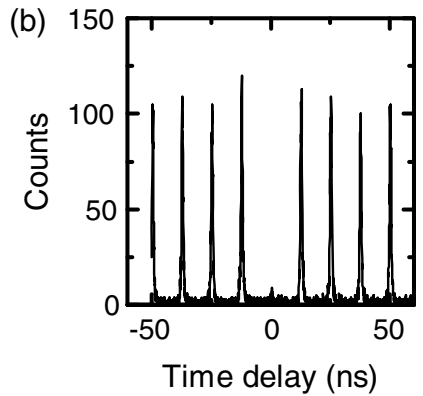

FIG. 4. (a) Rabi oscillation observed upon exciting UP1 and detecting LP1. (b) Measurement of the second-order coherence for exciting UP1 with a $\pi$ pulse and detecting LP1 yielding $g^{(2)}(0)=0.03 \pm 0.01$. 
UP1 with 16-ps pulses at a detuning of $\Delta=4.8 \mathrm{~g}$. Clear Rabi oscillations are observed and fitted [red line in Fig. 4(a)] using a damped oscillation superimposed with a very weak background that is linear with the excitation power because of a finite leakage of the excitation laser into the detection channel. This power-dependent damping is a direct consequence of the polariton-phonon interaction. With increasing laser power, the polariton levels hybridize, mixing higher rungs with UP1. This process increases the number of available damping channels via the polaritonphonon coupling and results in a damped oscillation.

To investigate single-photon generation in this configuration, we measure $g^{(2)}(0)$ while exciting UP1 with a $\pi$ pulse and detecting LP1 emission. The result of this experiment is presented in Fig. 4(b), and it shows almost perfect antibunching. By integrating the area of the peaks, we obtain a value of $g^{(2)}(0)=0.04 \pm 0.01$. Fitting the data with a series of Gaussian peaks is a little less sensitive to noise from the dark counts of the detectors and yields $g^{(2)}(0)=0.03 \pm 0.01$ (see Supplemental Material Ref. [32] for the fit and a closer examination of the data). These results rival the best values obtained from QDs while gaining advantages provided by the optical cavity (e.g., the very short lifetime that enables a high photon generation rate and the potential for integration into on-chip optical networks). Hence, our findings clearly demonstrate the potential of exploiting the efficient exciton-phonon coupling of strongly coupled QD-cavity systems for single-photon generation.

\section{SUMMARY AND CONCLUSIONS}

The presence of phonons represents one of the key distinguishing features of the solid state. Our investigations have laid the groundwork for understanding their role in the dynamics of strongly coupled systems. We have mapped out the detuning-dependent polariton lifetime of a strongly coupled QD-cavity system and extracted the spectrum of the polariton-to-phonon coupling for QD-cavity detunings up to $11 \mathrm{~g}$. We have shown that in order to obtain a high-fidelity photon blockade, a pulse length must be chosen that depends on the QD-cavity detuning. Finally, we have achieved direct coherent control of polariton states and provided an unprecedented demonstration of how the efficient coupling to phonons can be exploited for high-fidelity single-photon generation. Our findings suggest that the influence of the efficient coupling to phonons should be considered for all applications of strongly coupled solid-state systems. Furthermore, we expect that our contribution towards understanding strongly coupled systems in the solid state will play a vital role in utilizing QD-cavity platforms for novel physics such as higher-order nonclassical light generation [3].

\section{ACKNOWLEDGMENTS}

We gratefully acknowledge financial support from the Air Force Office of Scientific Research, MURI Center for Multifunctional Light-Matter Interfaces Based on Atoms and Solids (Grant No. FA9550-12-1-0025) and support from the Army Research Office (Grant No. W911NF1310309). K. M. acknowledges support from the Alexander von Humboldt Foundation. K. A.F. and Y. A.K. acknowledge support from SGF and the Department of Defense through NDSEG. K. G. L. acknowledges support from the Swiss National Science Foundation. V. B. acknowledges support from the National Science Foundation (Grant No. DGE-114747). K. M. and K. A. F. contributed equally to this work.

[1] A. Faraon, I. Fushman, D. Englund, N. Stoltz, P. Petroff, and J. Vuckovic, Coherent Generation of Non-classical Light on a Chip via Photon-Induced Tunnelling and Blockade, Nat. Phys. 4, 859 (2008).

[2] T. Volz, A. Reinhard, M. Winger, A. Badolato, K. J. Hennessy, E. L. Hu, and A. Imamoglu, Ultrafast All-Optical Switching by Single Photons, Nat. Photonics 6, 605 (2012).

[3] C. Sánchez-Muñoz, E. del Valle, A. González Tudela, K. Müller, S. Lichtmannecker, M. Kaniber, C. Tejedor, J. J. Finley, and F. P. Laussy, Emitters of N-Photon Bundles, Nat. Photonics 8, 550 (2014).

[4] C. J. Hood, M. S. Chapman, T. W. Lynn, and H. J. Kimble, Real-Time Cavity QED with Single Atoms, Phys. Rev. Lett. 80, 4157 (1998).

[5] J. Mckeever, A. Boca, A. D. Boozer, J. R. Buck, and H. J. Kimble, Experimental Realization of a One-Atom Laser in the Regime of Strong Coupling, Nature 425, 268 (2003).

[6] H. Mabuchi and A.C. Doherty, Cavity Quantum Electrodynamics: Coherence in Context, Science 298, 1372 (2002).

[7] J. M. Fink, M. Goppl, M. Baur, R. Bianchetti, P. J. Leek, A. Blais, and A. Wallraff, Climbing the Jaynes-Cummings Ladder and Observing Its Nonlinearity in a Cavity QED System, Nature 454, 315 (2008).

[8] T. Niemczyk, F. Deppe, H. Huebl, E. P. Menzel, F. Hocke, M. J. Schwarz, J. J. Garcia-Ripoll, D. Zueco, T. Hummer, E. Solano, A. Marx, and R. Gross, Circuit Quantum Electrodynamics in the Ultrastrong-Coupling Regime, Nat. Phys. 6, 772 (2010).

[9] J. P. Reithmaier, G. Sek, A. Loffler, C. Hofmann, S. Kuhn, S. Reitzenstein, L. V. Keldysh, V. D. Kulakovskii, T. L. Reinecke, and A. Forchel, Strong Coupling in a Single Quantum Dot-Semiconductor Microcavity System, Nature 432, 197 (2004).

[10] A. Högele, S. Seidl, M. Kroner, K. Karrai, R. J. Warburton, B. D. Gerardot, and P. M. Petroff, Voltage-Controlled Optics of a Quantum Dot, Phys. Rev. Lett. 93, 217401 (2004).

[11] J. H. Prechtel, A. V. Kuhlmann, J. Houel, L. Greuter, A. Ludwig, D. Reuter, A. D. Wieck, and R. J. Warburton, Frequency-Stabilized Source of Single Photons from a Solid-State Qubit, Phys. Rev. X 3, 041006 (2013).

[12] A. V. Kuhlmann, J. Houel, A. Ludwig, L. Greuter, D. Reuter, A. D. Wieck, M. Poggio, and R. J. Warburton, 
Charge Noise and Spin Noise in a Semiconductor Quantum Device, Nat. Phys. 9, 570 (2013).

[13] K. Hennessy, A. Badolato, M. Winger, D. Gerace, M. Atatüre, S. Gulde, S. Fält, E. L. Hu, and A. Imamoğlu, Quantum Nature of a Strongly Coupled Single Quantum Dot-Cavity System, Nature 445, 896 (2007).

[14] G. Reithmaier, S. Lichtmannecker, T. Reichert, P. Hasch, K. Muller, M. Bichler, R. Gross, and J. J. Finley, On-Chip Time Resolved Detection of Quantum Dot Emission Using Integrated Superconducting Single Photon Detectors, Sci. Rep. 3, 1901 (2013).

[15] M. Glässl, A. M. Barth, and V. M. Axt, Proposed Robust and High-Fidelity Preparation of Excitons and Biexcitons in Semiconductor Quantum Dots Making Active Use of Phonons, Phys. Rev. Lett. 110, 147401 (2013).

[16] P.-L. Ardelt, L. Hanschke, K. A. Fischer, K. Müller, A. Kleinkauf, M. Koller, A. Bechtold, T. Simmet, J. Wierzbowski, H. Riedl, G. Abstreiter, and J. J. Finley, Dissipative Preparation of the Exciton and Biexciton in Self-Assembled Quantum Dots on Picosecond Time Scales, Phys. Rev. B 90, 241404 (2014).

[17] J. H. Quilter, A. J. Brash, F. Liu, M. Glässl, A. M. Barth, V. M. Axt, A. J. Ramsay, M. S. Skolnick, and A. M. Fox, Phonon-Assisted Population Inversion of a Single InGaAs/GaAs Quantum Dot by Pulsed Laser Excitation, Phys. Rev. Lett. 114, 137401 (2015).

[18] M. Winger, T. Volz, G. Tarel, S. Portolan, A. Badolato, K. J. Hennessy, E. L. Hu, A. Beveratos, J. Finley, V. Savona, and A. Imamoğlu, Explanation of Photon Correlations in the Far-off-Resonance Optical Emission from a Quantum-Dot Cavity System, Phys. Rev. Lett. 103, 207403 (2009).

[19] M. Calic, P. Gallo, M. Felici, K. A. Atlasov, B. Dwir, A. Rudra, G. Biasiol, L. Sorba, G. Tarel, V. Savona, and E. Kapon, Phonon-Mediated Coupling of InGaAs/GaAs Quantum-Dot Excitons to Photonic Crystal Cavities, Phys. Rev. Lett. 106, 227402 (2011).

[20] U. Hohenester, A. Laucht, M. Kaniber, N. Hauke, A. Neumann, A. Mohtashami, M. Seliger, M. Bichler, and J. J. Finley, Phonon-Assisted Transitions from Quantum Dot Excitons to Cavity Photons, Phys. Rev. B 80, 201311 (2009).

[21] J. Suffczynski, A. Dousse, K. Gauthron, A. Lemaitre, I. Sagnes, L. Lanco, J. Bloch, P. Voisin, and P. Senellart, Origin of the Optical Emission within the Cavity Mode of Coupled Quantum Dot-Cavity Systems, Phys. Rev. Lett. 103, 027401 (2009).

[22] K. Müller, A. Rundquist, K. A. Fischer, T. Sarmiento, K. G. Lagoudakis, Y. A. Kelaita, C. Sánchez-Muñoz, E. del Valle, F. P. Laussy, and J. Vučković, Coherent Generation of Nonclassical Light on Chip via Detuned Photon Blockade, Phys. Rev. Lett. 114, 233601 (2015).

[23] Y. Akahane, T. Asano, B.-S. Song, and S. Noda, High-Q Photonic Nanocavity in a Two-Dimensional Photonic Crystal, Nature 425, 944 (2003).

[24] F. P. Laussy, E. del Valle, M. Schrapp, A. Laucht, and J. J. Finley, Climbing the Jaynes-Cummings Ladder by Photon Counting, J. Nanophoton. 6, 061803 (2012).
[25] D. Englund, A. Faraon, I. Fushman, N. Stoltz, P. Petroff, and J. Vuckovic, Controlling Cavity Reflectivity with a Single Quantum Dot, Nature 450, 857 (2007).

[26] D. Englund, D. Fattal, E. Waks, G. Solomon, B. Zhang, T. Nakaoka, Y. Arakawa, Y. Yamamoto, and J. Vučković, Controlling the Spontaneous Emission Rate of Single Quantum Dots in a Two-Dimensional Photonic Crystal, Phys. Rev. Lett. 95, 013904 (2005).

[27] M. Kaniber, A. Laucht, T. Hürlimann, M. Bichler, R. Meyer, M.-C. Amann, and J. J. Finley, Highly Efficient SinglePhoton Emission from Single Quantum Dots within a TwoDimensional Photonic Band-Gap, Phys. Rev. B 77, 073312 (2008).

[28] U. Hohenester, Cavity Quantum Electrodynamics with Semiconductor Quantum Dots: Role of Phonon-Assisted Cavity Feeding, Phys. Rev. B 81, 155303 (2010).

[29] S. Hughes, P. Yao, F. Milde, A. Knorr, D. Dalacu, K. Mnaymneh, V. Sazonova, P. J. Poole, G. C. Aers, J. Lapointe, R. Cheriton, and R. L. Williams, Influence of Electron-Acoustic Phonon Scattering on Off-Resonant Cavity Feeding within a Strongly Coupled Quantum-Dot Cavity System, Phys. Rev. B 83, 165313 (2011).

[30] C. Roy and S. Hughes, Influence of Electron AcousticPhonon Scattering on Intensity Power Broadening in a Coherently Driven Quantum-Dot Cavity System, Phys. Rev. X 1, 021009 (2011).

[31] G. Reithmaier, F. Flassig, P. Hasch, S. Lichtmannecker, K. Müller, J. Vuckovic, R. Gross, M. Kaniber, and J. J. Finley, A Carrier Relaxation Bottleneck Probed in Single InGaAs Quantum Dots Using Integrated Superconducting Single Photon Detectors, Appl. Phys. Lett. 105, 081107 (2014).

[32] See Supplemental Material at http://link.aps.org/ supplemental/10.1103/PhysRevX.5.031006, which includes Refs. [33, 34], for a detailed description of the employed methods and models, theoretical derivations, details on the simulations, and additional supporting measurements and simulations.

[33] Y. Akahane, T. Asano, B.-S. Song, and S. Noda, Fine-Tuned High-Q Photonic-Crystal Nanocavity, Opt. Express 13, 1202 (2005).

[34] M. Minkov and V. Savona, Automated Optimization of Photonic Crystal Slab Cavities, Sci. Rep. 4, 5124 (2014).

[35] A. Reinhard, T. Volz, M. Winger, A. Badolato, K. J. Hennessy, E. L. Hu, and A. Imamoglu, Strongly Correlated Photons on a Chip, Nat. Photonics 6, 93 (2011).

[36] R. J. Glauber, The Quantum Theory of Optical Coherence, Phys. Rev. 130, 2529 (1963).

[37] R. Loudon, The Quantum Theory of Light (Oxford Science Publications, Oxford, 2000).

[38] C. Santori, M. Pelton, G. Solomon, Y. Dale, and Y. Yamamoto, Triggered Single Photons from a Quantum Dot, Phys. Rev. Lett. 86, 1502 (2001).

[39] All pulse lengths are quoted as full width at half maximu throughout the text.

[40] J. R. Johansson, P. D. Nation, and F. Nori, QuTiP 2: A Python Framework for the Dynamics of Open Quantum Systems, Comput. Phys. Commun. 184, 1234 (2013). 